\title{
Produção de fitomassa de Sálvia officinalis L. cultivada sob malhas coloridas e doses de esterco avícola
}

\section{Production of phytomass of Salvia officinalis L. cultivated under colored fabrics and doses of poultry manure}

\author{
Girlene Santos de Souza ${ }^{1 *}$; Janderson do Carmo Lima ${ }^{2}$; Anacleto Ranulfo dos Santos ${ }^{3}$; Uasley Caldas de Oliveira ${ }^{4}$; Mariana \\ Nogueira Bezerra ${ }^{5}$
}

\begin{abstract}
Resumo: Objetivou-se avaliar o efeito da qualidade de luz na produção de fitomassa de plantas de Sálvia Oficcinalis L. cultivada sob malhas coloridas e doses de esterco avícola. $\mathrm{O}$ experimento foi conduzido em área experimental da Universidade Federal do Recôncavo da Bahia, onde as plantas foram dispostas em diferentes ambientes de luz e cinco doses de esterco avícola $\left(0 ; 60 ; 120 ; 180\right.$ e $\left.240 \mathrm{Kg} \mathrm{ha}^{-1}\right)$. Aos 90 dias foram aferidas medidas não lineares: área foliar e peso de matéria seca e medidas lineares: número de folhas e altura das plantas. Houve uma interação significativa entre os ambientes de luz e as doses de esterco utilizadas para as variáveis área foliar (AF) e razão de massa foliar (RMF), encontrando os maiores valores de RMF com incremento de $72 \%$ e AF com $66 \%$ nas plantas submetidas as maiores doses de esterco. Analisando o efeito isolado dos ambientes de luz a malha vermelha teve destaque em todas as variáveis com exceção do número de folhas que não foram significativos. O uso de malhas coloridas em interação com doses de esterco avícola proporcionam expressivas respostas ao crescimento e desenvolvimento da Salvia officinalis.
\end{abstract}

Palavras-chave: Ambientes de luz; Cama de frango; Fertilização Orgânica; Planta medicinal.

Abstract: Objective to evaluate the effect of light quality on production of phytomass of Sálvia Oficcinalis L. cultivated under colored fabrics and doses of poultry manure. The experiment was conducted in an experimental area of the Federal University of Recôncavo of Bahia, where the plants were arranged in different light environments and five doses of poultry manure $(0 ; 60$; 120; 180 and $240 \mathrm{Kg} \mathrm{ha}^{-1}$ ). To 90 days were measured non-linear measures: leaf area and dry matter weight and linear measures: number of leaves and the height of the plants. There was a significant interaction between light environments and the doses of manure used for variables leaf area (AF) and foliar mass ratio (RMF), finding the highest values of RMF with $72 \%$ increment and AF with $66 \%$ in plants submitted the largest doses of manure. Analyzing the isolated effect of light red mesh environments had featured in all the variables with the exception of the number of sheets that were not significant. It is concluded that the use of colored meshes in interaction with doses of poultry manure provide significant responses to the growth and development of Salvia officinalis.

Key words: Light environments; Chicken bed; Organic Fertilization; Medicinal plant.

\footnotetext{
*Autor para correspondência

Recebido para publicação em 19/04/2016; aprovado em 02/02/2017

${ }^{1}$ Professora Associada 1, Universidade federal do Recôncavo da Bahia, Cruz das Almas, Bahia; girlenessouza50@gmail.com

${ }^{2}$ Mestre em Solos e Qualidade de Ecossistemas, Universidade federal do Recôncavo da Bahia, Cruz das Almas, Bahia; janderson_ufrb.yahoo.com.br

${ }^{3}$ Professor Titular, Universidade federal do Recôncavo da Bahia, Cruz das Almas, Bahia; anacleto@ufrb.edu.br

${ }^{4}$ Graduando em Agronomia, Universidade federal do Recôncavo da Bahia, Cruz das Almas, Bahia; uasley@ gmail.com

${ }^{5}$ Graduanda em Engenharia Florestal, Universidade federal do Recôncavo da Bahia, Cruz das Almas, Bahia; mnbflorestal@gmail.com
} 


\section{INTRODUÇÃO}

A Sálvia officinalis L., pertencente à família Lamiaceae, é originária do mediterrâneo e tem se difundido principalmente, na região Sul do Brasil. Conhecida popularmente como sálvia, é uma planta aromática usada, principalmente, para fins medicinais e alimentícios que apresenta elevada expressão de metabolitos secundários uteis, tais como: compostos terpenóides e derivados fenólicos (BEN FARHAT et al., 2013; ROBY et al., 2013), sendo muito utilizada pela indústria farmacêutica e de condimentos. Para o cultivo comercial em larga escala da espécie, ainda há necessidade de desenvolver trabalhos que identifiquem um manejo fitotécnico mais adequado, conforme os casos da adubação e do controle fisiológico das plantas, visando maior produção de biomassa com qualidade.

As constantes alterações climáticas e as exigências do mercado por produtos de qualidade têm contribuído para que produtores de plantas medicinais busquem tecnologias de produção que permitem produzir em condições ambientais adversas (altas temperaturas, radiação solar, ventos). No cultivo protegido, uma das alternativas é a utilização de filtros espectrais, que transmitem seletivamente certos comprimentos de ondas (COSTA et al., 2012), os quais resultam em alterações nas funções fisiológicas, na morfologia, no crescimento e desenvolvimento das plantas, como resposta à adaptação a uma condição ambiental diferenciada de luminosidade (TSORMPATSIDIS et al., 2008).

As malhas fotoconversoras alteram o espectro de luz transmitido, dentre elas, a malha vermelha, que apresenta maior transmitância em comprimentos de onda acima de 590 $\mathrm{nm}$ (vermelho) e um pico menor em torno de $400 \mathrm{~nm}$ (violeta), reduzindo ondas azuis, verdes e amarelas. Sendo assim, a luz vermelha atua no desenvolvimento da estrutura fotossintética, favorecendo o crescimento das plantas (SHAHAK et al., 2008). A luz vermelha atua no desenvolvimento da estrutura fotossintética das plantas, o que pode aumentar o acúmulo de amido em algumas espécies, pela inibição da translocação de assimilados para fora das folhas (SAEBO; MORTENSEN, 1996).

A luz azul permite alterar o crescimento, o desenvolvimento e a aclimatação às condições ambientais das plantas (TAIZ; ZEIGER, 2013). Assim, a utilização de malhas com transmissão seletiva de radiação UV pode oferecer um caminho para aumentar a produção de metabólitos secundários em plantas medicinais e aromáticas. Contudo, tem sido demandados estudos sobre os efeitos da radiação UV no crescimento, no desenvolvimento e na produção de fitomassa e metabólitos das plantas (TSORMPATSIDIS et al., 2008).

Essas malhas têm como finalidade combinar a proteção física com a filtração diferencial da radiação solar, para promover respostas fisiológicas específicas que são reguladas pela luz (BRANT et al., 2009).ou seja as malhas coloridas têm a característica de alterar a qualidade espectral da radiação, permitindo assim a manipulação do crescimento e desenvolvimento de plantas (NOMURA et al., 2009).

Outro fator limitante para o desenvolvimento e produção de plantas medicinais de uma maneira em geral é o manejo do solo, sendo este um fator fundamental para obtenção de maior sucesso de produção através do fornecimento adequado de uma fonte de nutrientes, principalmente no que diz respeito à distribuição dos mesmos no solo, fator esse primordial para evitar o desequilíbrio nutricional de algumas culturas.

A matéria orgânica no solo é considerada uma das principais fontes de energia e nutrientes ao sistema, capaz de manter a produtividade dos solos. Entre outros benefícios da matéria orgânica, destacam-se a melhoria das condições físicas, químicas e biológicas do solo e o fornecimento de energia para o crescimento microbiano (SILVA; RESCK, 1997) o que reflete em maior ciclagem de nutrientes, aumento da CTC do solo e infiltração de água, diminuindo as perdas por erosão e melhorando o solo para o preparo (PAES et al., 1996). O uso de matéria orgânica, de origem vegetal ou animal, por exemplo, promove melhoria nas propriedades edáficas do solo sob manejo orgânico de produção (CARDOZO et al., 2008; LOSS, 2008). Este fato, aliado ao alto custo dos adubos minerais, tem levado ao aumento de estudos para o aproveitamento da cama de aves como fertilizante orgânico (LANA et al., 2010), em virtude de sua riqueza em nutrientes (COSTA et al., 2009). O uso de adubos orgânicos como cama de aves, tornou-se alternativa interessante, devido aos centros avícolas e por possuir preço inferior aos adubos sintéticos (COSTA et al., 2009), sendo a sua utilização na agricultura uma alternativa viável para redução da poluição ambiental, além de ser uma fonte de nutrientes em potencial para agricultura orgânica do País.

O presente trabalho teve como objetivo avaliar o efeito da qualidade de luz na produção de fitomassa de plantas de Sálvia Oficcinalis L. cultivadas sob malhas coloridas e doses de esterco avícola.

\section{MATERIAL E MÉTODOS}

O experimento foi conduzido entre abril e agosto de 2014, na Universidade Federal do Recôncavo da Bahia (UFRB), em Cruz das Almas. As mudas foram produzidas pelo método de reprodução sexuada, e com 15 dias medindo em média $10 \mathrm{~cm}$ de comprimento foram transplantadas para vasos com capacidade de $3 \mathrm{dm}^{3}$, contendo como substrato solo e areia lavada na proporção $2: 1$, onde permaneceram em casa de vegetação por 20 dias para aclimatação.

O solo utilizado como substrato foi o Latossolo Amarelo Coeso, coletado em área de pastagem natural, do campus da UFRB, na camada de $0-20 \mathrm{~cm}$. Posteriormente foi realizado análise química do mesmo e os valores com as características químicas são apresentando na tabela 1.

Tabela 1. Características químicas do solo da área experimental para o cultivo da Sálvia em Cruz das Almas, Bahia

\begin{tabular}{|c|c|c|c|c|c|c|c|c|c|c|c|c|}
\hline \multirow[t]{2}{*}{ Prof. } & \multirow[t]{2}{*}{$\mathrm{pH}$} & \multicolumn{2}{|r|}{$P$} & \multirow{2}{*}{$\begin{array}{c}\mathrm{K} \\
\mathrm{mg} \mathrm{d \textrm {dm } ^ { - 3 }}\end{array}$} & \multirow[t]{2}{*}{$\mathrm{Ca}$} & \multirow[t]{2}{*}{$\mathrm{Mg}$} & \multirow{2}{*}{$\begin{array}{c}\mathrm{Al} \\
\mathrm{Cmol}\end{array}$} & \multirow[t]{2}{*}{$\mathrm{H}+\mathrm{Al}$} & \multirow[t]{2}{*}{ S } & CTC & \multirow[t]{2}{*}{ V } & \multirow[t]{2}{*}{ M.O } \\
\hline & & $(\mathrm{cm})$ & $\mathrm{H}_{2} \mathrm{O}$ & & & & & & & $\mathrm{g} /$ & & \\
\hline $0,0-20$ & 5,38 & & 16 & 47 & 1,0 & 0,7 & 0,2 & 2,70 & 1,95 & 4,65 & 41,9 & 16,50 \\
\hline
\end{tabular}

Foi feita uma adubação de plantio utilizando ureia, super triplo e cloreto de potássio nas doses de 90,100 e $60 \mathrm{~kg} \mathrm{ha}^{-1}$ respectivamente em todos os vasos.
Utilizou-se cinco doses de esterco avícola: $0 ; 60 ; 120$; 180 e $240 \mathrm{Kg} \mathrm{ha}^{-1}$ sendo distribuído conforme cálculo de adubação por vaso de cada tratamento respectivamente, e 3 ambientes de luz: malha vermelha, e azul, com $50 \%$ de 
sombreamento e a pleno sol. As plantas foram mantidas por 90 dias sob os tratamentos em cinco repetições. Foi utilizado um esquema fatorial $5 \times 3$ (doses de esterco e ambientes de luz) totalizando 75 unidades experimentais, em delineamento inteiramente casualizado.

Foram aferidas medidas biométricas lineares e não lineares. As medidas biométricas não lineares foram determinadas a partir dos valores de área foliar e do peso da matéria seca da planta (folha, caule e raiz). A área foliar total por planta foi medida utilizando-se medidor de área foliar portátil "AM300 Área Meter" da marca ADC. As medidas biométricas lineares: número de folhas e altura da planta foram avaliados antes da colheita da planta e esta análise foi determinada com o auxílio de uma régua. Também determinou-se a razão massa foliar (RMF), de acordo com Benincasa (2004).

Após a colheita, os componentes das plantas foram separados, e desidratados em estufa de circulação de ar forçada a $65^{\circ} \pm 5^{\circ} \mathrm{C}$ por 72 horas, até alcançar massa constante, para posterior aferição de massa de matéria seca em balança analítica de precisão centesimal.

Os resultados obtidos foram submetidos à análise de variância utilizando-se o programa estatístico SISVAR (FERREIRA, 2008). Em função do nível de significância e foi aplicado o teste de Tukey a $5 \%$ de probabilidade e de regressão para identificar o efeito das malhas coloridas e das doses de esterco avícola, respectivamente.

\section{RESULTADOS E DISCUSSÃO}

Houve interação significativa entre as doses de esterco avícola utilizadas e os ambientes de luz (Tabela 2), para as variáveis área foliar (AF) e razão de massa foliar (RMF).

Foram encontrados maiores valores de RMF e AF quando as plantas foram submetidas à maior dose de esterco avícola e sob malha vermelha, destacando a AF que apresentou um acréscimo de $66 \%$ em relação ao tratamento com $180 \mathrm{Kg} \mathrm{ha}^{-1}$ e também sob a mesma malha. Salienta-se que essa variável é de extrema importância, pois representa a dimensão do aparelho fotossintetizante (PEIXOTO, 2011).

Tabela 2. Desdobramento da interação para as variáveis Area foliar (AF) e Razão de massa foliar (RMF), entre doses de esterco avícola dentro de cada nível de ambiente de luz, das plantas de Sálvia, Cruz das Almas-Bahia

\begin{tabular}{cccc}
\hline & & $\mathbf{A F}\left(\mathbf{c m}^{2}\right)$ & \\
\hline Tratamentos & Pleno sol & Malha azul & Malha vermelha \\
$0 \mathrm{Kg} \mathrm{ha}^{-1}$ & $200,1 \mathrm{Aa}$ & $143,6 \mathrm{Aa}$ & $235,4 \mathrm{Ab}$ \\
$60 \mathrm{Kg} \mathrm{ha}^{-1}$ & $210,1 \mathrm{Aa}$ & $144,2 \mathrm{Aa}$ & $253,1 \mathrm{Ab}$ \\
$120 \mathrm{Kg} \mathrm{ha}^{-1}$ & $221,2 \mathrm{Aa}$ & $196,3 \mathrm{Aa}$ & $272,4 \mathrm{Ab}$ \\
$180 \mathrm{Kg} \mathrm{ha}^{-1}$ & $233,2 \mathrm{Aa}$ & $247,7 \mathrm{Aa}$ & $350,7 \mathrm{Ab}$ \\
$240 \mathrm{Kg} \mathrm{ha}^{-1}$ & $290,4 \mathrm{Ba}$ & $262,6 \mathrm{Ba}$ & $527,1 \mathrm{Aa}$ \\
\hline & & & $0,24 \mathrm{Ac}$ \\
\hline $0 \mathrm{Kg} \mathrm{ha}^{-1}$ & $0,24 \mathrm{Aa}$ & $0,26 \mathrm{Aa}$ & $0,29 \mathrm{Abc}$ \\
$60 \mathrm{Kg} \mathrm{ha}^{-1}$ & $0,25 \mathrm{Aa}$ & $0,28 \mathrm{Aa}$ & $0,31 \mathrm{Abc}$ \\
$\left.120 \mathrm{Kg} \mathrm{ha}^{-1}\right)$ & $0,29 \mathrm{Aa}$ & $0,36 \mathrm{Ab}$ \\
$180 \mathrm{Kg} \mathrm{ha}^{-1}$ & $0,27 \mathrm{Aa}$ & $0,30 \mathrm{Aa}$ & $0,50 \mathrm{Aa}$ \\
$240 \mathrm{Kg} \mathrm{ha}^{-1}$ & $0,31 \mathrm{Aa}$ & $0,32 \mathrm{Ba}$ & $0,29 \mathrm{Ba}$ \\
\hline
\end{tabular}

*letra minúscula para comparação entre colunas e maiúscula em linhas, pelo teste de Tukey a 5\% de probabilidade.

Costa et al. (2012) verificou um ganho de fitomassa seca de folhas nas plantas de hortelã-pimenta cultivadas sob malha vermelha o que pode estar relacionado ao incremento da área foliar que foi de $1.343,62 \mathrm{~cm}^{2}$ por planta. Entretanto, Chagas et al., (2010), encontraram resultados similares quando trabalhou com hortelã-japonesa cultivada sob diferentes malhas.

A RMF definida pela relação entre a massa da matéria seca retida nas folhas e a massa da matéria seca retida na planta, principalmente quando a matéria prima são as folhas, como foi o caso da sálvia, fazendo o mesmo efeito comparativo entre as maiores doses de esterco avícola utilizadas dentro da malha vermelha. Percebeu-se para essa variável um incremento de $72 \%$, destacando mais uma vez o efeito positivo encontrado nessa interação.

Costa et al. (2008) estudando diferentes tipos e doses de adubação orgânica no crescimento e na composição química do óleo essencial elixir paregórico observou-se um incremento da área foliar com as doses de adubação, atingindo valores máximos de $6084 \mathrm{~cm}^{2}$ com as doses de 9,6 $\mathrm{kg} \mathrm{m} \mathrm{m}^{2}$ de esterco avícola, já para a RMF ocorreu o inverso chegando a encontrar maiores valores nos tratamentos sem a adição de adubo orgânico ocorrendo assim uma diminuição significativa com o aumento da dose.

Brant et al., (2009) trabalhando com plantas de melissa cultivadas sob malhas fotoconversoras, não verificaram diferença significativa para RMF o que difere dos resultados encontrados por Martins (2008) que no cultivo de Ocimum gratissimum L. em ambientes de pleno sol e malha azul demonstraram os menores valores do que em plantas cultivadas nas malhas preta e vermelha, onde o mesmo autor afirma que isso demonstra que tratamentos sob pleno sol ou sob malha azul alocam menos fotoassimilados para as folhas em relação aos demais tratamentos.

No cultivo de manjericão sob malha aluminizada, Paulus et al. (2016) observaram maior área foliar $\left(1840,22 \mathrm{~cm}^{2}\right)$ que o cultivo sob malha preta, vermelha e pleno sol. Os menores valores de área foliar verificados nas plantas crescidas sob malha vermelha, preta e sob pleno sol indicam que ocorreu menor alocação de fotoassimilados para as folhas, ao contrário do que ocorreu neste trabalho onde maiores valores de AF forma observados nas plantas cultivadas sob a malha vermelha.

Estudando o efeito isolado dos ambientes de luz (Tabela 3 ), verificou-se que as plantas crescidas sob malha vermelha apresentaram valores significativos para as variáveis altura (ALT), massa da matéria seca de folhas (MSF), massa da matéria seca do caule (MSC) e massa da matéria seca de raiz (MSR) em relação aos demais tratamentos. O NF não apresentou significância para esses tratamentos. 
Tabela 3. Altura (ALT), massa da matéria seca de folhas (MSF), caule (MSC) e raiz (MSR) de plantas de Sálvia cultivadas sob diferentes ambientes de luz, Cruz das Almas-Bahia

\begin{tabular}{ccccc}
\hline Ambientes de Luz & $\begin{array}{c}\text { ALT } \\
(\mathbf{c m})\end{array}$ & $\begin{array}{c}\text { MSF } \\
(\mathbf{g})\end{array}$ & $\begin{array}{c}\text { MSC } \\
(\mathbf{g})\end{array}$ & $\begin{array}{c}\text { MSR } \\
(\mathbf{g})\end{array}$ \\
\hline Pleno Sol & $21,15 \mathrm{c}$ & $2,59 \mathrm{~b}$ & $0,94 \mathrm{~b}$ & $5,70 \mathrm{a}$ \\
Malha Azul & $24,39 \mathrm{~b}$ & $2,15 \mathrm{~b}$ & $1,05 \mathrm{~b}$ & $4,08 \mathrm{~b}$ \\
Malha vermelha & $28,61 \mathrm{a}$ & $3,69 \mathrm{a}$ & $1,64 \mathrm{a}$ & $5,10 \mathrm{a}$ \\
\hline CV $(\%)$ & 16,33 & 13,58 & 23,68 & 24,82 \\
\hline
\end{tabular}

Paulus et al. (2016) verificaram que as plantas de manjericão apresentaram alterações no crescimento em função das diferentes cores das malhas de sombreamento, ou seja, plantas crescidas sob malha aluminizada apresentaram maior crescimento em altura $(66,58 \mathrm{~cm})$ em relação as plantas crescidas a pleno sol que apresentaram a menor altura, em torno de $(53,01 \mathrm{~cm})$.

Segundo Costa et al. (2012) a fitomassa total das plantas de hortelã-pimenta cultivadas a pleno sol e sob malha vermelha foi maior que a das demais, e o menor acúmulo ocorreu nas plantas cultivadas sob malha azul, enquanto que a fitomassa seca da raiz sob a malha vermelha e termorrefletora não diferiram entre si, porém, foram menores em comparação à massa seca de raiz de plantas cultivadas em pleno sol (COSTA et al., 2012)

Avaliando o efeito isolado das doses de esterco avícola utilizadas nas plantas de sálvia percebeu-se desempenho linear positivo para MSF e NF, apresentando siginificância á $1 \%$ em relação as demais doses utilizadas (Figura1 A e B).

Figura 1. A) Massa da matéria seca de folhas (MSF) e B) número de folhas de plantas de sálvia cultivadas sob doses de esterco avícola, Cruz das Almas-Bahia

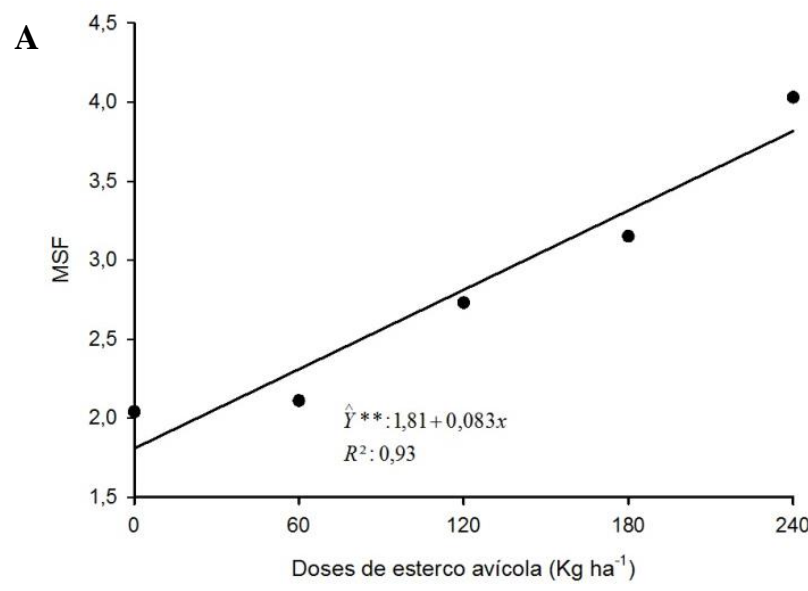

A maior produção de folhas ocorreu possivelmente pelo fato da adubação via esterco avícola proporcionar o maior fornecimento de nutrientes a cultura, com isso alcançando uma maior produção de folhas que consequentemente aumentou a atividade fotossintética da planta. Dados estes que corroboram com os obtidos por Silva et al. (2016) que observaram, em seu trabalho o aumento nos teores de nutrientes nos tecidos foliares do feijão-vagem em função de doses de adubação orgânica.

\section{CONCLUSÕES}

Os ambientes de luz promovem incrementos significativos nas variáveis altura e massa seca de folha, caule e raiz.

A qualidade de luz através das malhas fotoconversoras influenciou o acúmulo de fitomassa nas plantas de sálvia.

$\mathrm{O}$ comportamento da Salvia em relação às doses do esterco avícola apresentou-se linear tendo respostas significativas até a dose mais elevada estudada.

$\mathrm{O}$ uso de malhas coloridas em interação com doses de esterco avícola proporcionam expressivas respostas ao crescimento da Salvia officinalis.

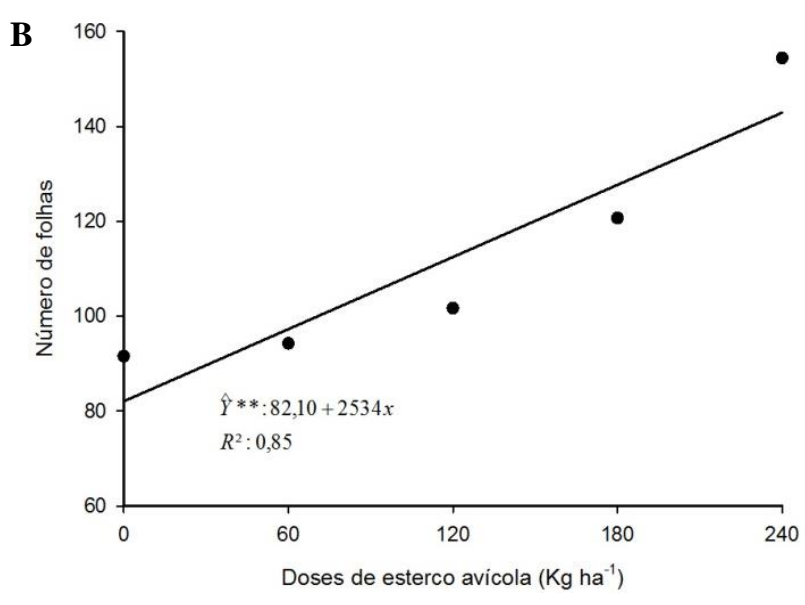

\section{REFERÊNCIAS}

BENINCASA, M. M. P. Análise de crescimento de plantas: noções básicas. Jaboticabal: FUNEP, p. 42, 2004.

BEN FARHAT, M.; LANDOULSI, A.; CHAOUCHHAMADA, R.; SOTOMAYOR, J. A.; JORDÁN, M. J. Characterization and quantification of phenolic compounds and antioxidant properties of Salvia species growing in different habitats. Industrial Crops and Products, v. 49, p. 904-914. 2013.

BRANT, R. da S.; PINTO , J.E.B.P.; Rosa, L.F.; ALBUQUERQUE, C.J.B.; FERI, P.H.; Corêa, R.M. Crescimento, teor e composição do óleo essencial de melissa cultivada sob malhas fotoconversoras. Ciência Rural, v. 39, p. 1401-1407, 2009.

CHAGAS, J. H.; PINTO, J. E. B. P.; BERTOLUCCI, S. K. V.; FERRAZ, E. de O.; BOTREL, P. P.; SANTOS, F. M. dos. Produção de biomassa seca em plantas de Mentha arvensis L. cultivada sob malhas fotoconversoras. Horticultura Brasileira, v.28, p.3422-3427, 2010. 
COSTA, L. C. B.; PINTO, J. E. B. P.; CASTRO, E. M.; Bertolucci, S. K. V.; Corrêa, R. M.; Reis, E. S.; Alves, P. B.; Niculau, E. S. Tipos e doses de adubação orgânica no crescimento, no rendimento e na composição química do óleo essencial de elixir paregórico. Ciência Rural, Santa Maria, v. 38, n. 8, p.2173-2180, nov. 2008.

COSTA, A. M.; BORGES, E. N.; SILVA, A. A.; NOLlA, A.; GUIMARÃES, E. C. Potencial de recuperação física de um Latossolo Vermelho, sob pastagem degradada, influenciado pela aplicação de cama de frango. Ciência e Agrotecnologia, Lavras, v. 33, p. 1991-1998, 2009.

COSTA, A. G.; CHAGAS, J. H.; PINTO, J. E. B. P.; Bertolucci, S. K. V. Crescimento vegetativo e produção de óleo essencial de hortelã-pimenta cultivada sob malhas. Pesquisa Agropecuária Brasileira, Brasília, v. 47, n. 4, p.534-540, abr. 2012.

FERREIRA, D. F.; SISVAR: um programa para análises e ensino de estatística. Revista Symposium, 6: 36-41, 2008.

LANA, R. M. Q.; ASSIS, D. F.; SILVA, A. A.; LANA, A. M. Q.; GUIMARÃES, E. C.; BORGES, E. N. Alterações na produtividade e composição nutricional de uma pastagem após segundo ano de aplicação de diferentes doses de cama de frango. Bioscience Journal, Uberlândia, v. 26, n. 2, p. 249256, 2010.

MARTINS, J. R.; ALVARENGA, A. A.; CASTRO, E. M.; PINTO, J. E. B. P.; SILVA, A. P. O. Avaliação do crescimento e do teor de óleo essencial em plantas de Ocimum gratissimum L. cultivadas sob malhas coloridas. Revista Brasileira de Plantas Medicinais, 10 (4): 102-107, 2008 .

NOMURA, E. S.; LIMA, J. D.; RODRIGUES, D. S.; GARCIA, V. A.; FUZITANI, E. J.; SILVA, S. H. M. Crescimento e produção de antúrio cultivado sob diferentes malhas de sombreamento. Ciência Rural, v.39, n.5, p.13941400, 2009.

PAES, J. M. V.; ANDREOLA, F.; BRITO, C. H.; LOUDES, E.G. Decomposição da palha de café em três tipos de solo e sua influência sobre a CTC e o pH. Revista Ceres, v.43, p. 337-342, 1996.
PAULUS D.; VALMORBIDA R; FERREIRA S. B; ZORZZI, I. C; NAVA G. A. Biomassa e composição do óleo essencial de manjericão cultivado sob malhas fotoconversoras e colhido em diferentes épocas. Horticultura Brasileira, v 34, n. 1, p. 46-53, 2016.

PEIXOTO, C. P.; CRUZ. T. V.; PEIXOTO, M. F. S. P. Análise quantitativa do crescimento de plantas: conceitos e prática. Enciclopédia biosfera, v.7, n.13; p. 51-76. 2011.

ROBY, M. H. H.; SARHAN, M. A.; SELIM, K. A. H.; KHALEL, K. I. Evaluation of antioxidant activity, total phenols and phenolic compounds in thyme (Thymus vulgaris L.), sage (Salvia officinalis L.), and marjoram (Origanum majorana L.) extracts. Industrial Crops and Products, v. 43, p. 827-831. 2013.

SAEBO, A.; MORTENSEN, L. M. The influence of elevated $\mathrm{CO}_{2}$ concentration on growth of seven grasses and one clover species in a cool maritime climate. Acta Agricultura e Scandinavia Section BSoil and Plant Science, v.46, p.49-54, 1996.

SHAHAK, Y. Photo-selective netting for improved performance of horticultural crops. A review of ornamental and vegetable studies carried out in Israel. Acta Horticulture, n.770, p.161-168, 2008.

SILVA, J. E.; RESCK, D. V. S. Matéria orgânica do solo. In: VARGAS, M. A. T.; HUNGRIA, M. Biologia dos solos dos Cerrados. Planaltina, Embrapa, p.467-524. 1997.

SILVA, I. C. M.; SILVA, J. G.; SANTOS, B. G. F. L.; DANTAS, M. V.; LIMA, T. S. Influência da adubação orgânica no desenvolvimento do feijão-vagem em diferentes níveis de água de irrigação Revista Verde de Agroecologia e Desenvolvimento Sustentável, v.11, n. ${ }^{\circ}$ 5, p. 01-07, 2016.

TAIZ, L.; ZEIGER, E. Fisiologia Vegetal. 3. ed. Porto Alegre: Artmed, 2013. 719p.

TSORMPATSIDIS, E.; HENBEST, R. G. C.; DAVIS, F. J.; BATTEYA, N. H.; HADLEYA, P.; WAGSTAFFE, A. UV irradiance as a major influence on growth, development and secondary products of commercial importance in Lollo Rosso lettuce 'Revolution' grown under polyethylene films. Environmental and Experimental Botany, v.63, p.232-239, 2008. 